\title{
[Ne III]/[O II] as an oxygen abundance indicator in the $H \|$ regions and $H \|$ galaxies
}

\author{
F. Shi, G. Zhao, and Y. C. Liang \\ National Astronomical Observatories, Chinese Academy of Sciences, 20A Datun Road, Chaoyang District, Beijing 100012 , \\ PR China \\ e-mail: fshi@bao.ac.cn
}

Received 29 January 2007 / Accepted 20 August 2007

\section{ABSTRACT}

\begin{abstract}
Aims. To calibrate the relationship between Ne3O2 (Ne3O2 = $\log ([\mathrm{Ne}$ III] $\lambda 3869 /[\mathrm{O}$ II $] \lambda 3727)$ ) and oxygen abundances, we present a sample of $\sim 3000$ H II galaxies from the Sloan Digital Sky Survey (SDSS) data release four. They are associated with a sample from the literature intended to enlarge the oxygen abundance region.

Methods. We calculated the electron temperatures $\left(T_{\mathrm{e}}\right)$ of 210 galaxies in the SDSS sample with the direct method, and $T_{\mathrm{e}}$ of the other 2960 galaxies in SDSS sample calculated with an empirical method. Then, we use a linear least-square fitting to calibrate the $\mathrm{Ne} 3 \mathrm{O} 2$ oxygen abundance indicator.

Results. It is found that the $\mathrm{Ne} 3 \mathrm{O} 2$ estimator follows a linear relation with $12+\log (\mathrm{O} / \mathrm{H})$ that holds for the whole abundance range covered by the sample, from approximately 7.0 to 9.0 . The best linear relationship between the $\mathrm{Ne} 3 \mathrm{O} 2$ and the oxygen abundance is calibrated. The dispersion between oxygen abundance and $\mathrm{Ne} 3 \mathrm{O} 2$ index in the metal rich galaxies may come partly from the moderate depletion of oxygen onto grains. The $\mathrm{Ne} 3 \mathrm{O} 2$ method has the virtue of being single-valued and not affected by internal reddening. As a result, the $\mathrm{Ne} 3 \mathrm{O} 2$ method can be a good metallicity indicator in the $\mathrm{H}$ II regions and $\mathrm{H}$ II galaxies, especially in high-redshift galaxies.
\end{abstract}

Key words. Galaxy: abundances - galaxies: ISM - H II regions

\section{Introduction}

Those hydrogen II galaxies with strong emission lines are important probes for the formation and evolution of galaxies. Their spectra contain the much important information needed to determine the star formation rate, initial mass function, element abundance, etc. (Stasińska \& Leitherer 1996; Kennicutt 1998; Contini et al. 2002). The heavy element abundance is a key parameter for the formation and evolution of a galaxy. Oxygen is the important element that is easily and reliably determined since all the most important ionization stages can be observed. The oxygen abundance from the measurement of electron temperature from [O III $] \lambda \lambda 4959,5007 /[\mathrm{O} \mathrm{III}] \lambda 4363$ is one of the most reliable methods. But [O III] $] 4363$ is usually weak in the low metallicity galaxies, and there are often large errors when measuring this line. In high metallicity galaxies, [O III] $] 4363$ are hardly even observable.

Instead of the $T_{\mathrm{e}}$ method, strong line methods, such as the $R_{23}{ }^{1}, P^{2}, N 2^{3}$, or O3N2 ${ }^{4}$ methods, are used widely (Pagel et al. 1979; Kobulnicky et al. 1999; Pilyugin et al. 2001; Charlot \& Longhetti 2001; Denicoló et al. 2002; Pettini \& Pagel 2004; Tremonti et al. 2004; Liang et al. 2006). The $R_{23}$ and $P$ methods suffer the double-valued problem, requiring some assumption or rough a priori knowledge of a galaxy's metallicity in order to locate it on the appropriate branch of the relation. The N2- and $\mathrm{O} 3 \mathrm{~N} 2$ methods are monotonic, but the reasons for this are not purely physical. It is partly due to the N/O ratio increases on

\footnotetext{
${ }^{1} R_{23}=([\mathrm{O}$ II $] \lambda 3727+[\mathrm{O}$ III $] \lambda \lambda 4959,5007) / \mathrm{H} \beta$.

${ }^{2} P=[\mathrm{O}$ III $] \lambda \lambda 4959,5007 /([\mathrm{O}$ II $] \lambda 3727+[\mathrm{O}$ III $] \lambda \lambda 4959,5007)$.

${ }^{3} \mathrm{~N} 2=\log ([\mathrm{N} \mathrm{II}] \lambda 6583 / \mathrm{H} \alpha)$.

${ }^{4} \mathrm{O} 3 \mathrm{~N} 2=\log (([\mathrm{O} \mathrm{III}] \lambda 5007 / \mathrm{H} \beta) /([\mathrm{N} \mathrm{II}] \lambda 6583 / \mathrm{H} \alpha))$.
}

average with the increase in metallicity (Stasińska 2006; Shi et al. 2006). Besides, calibrations of the O3N2 and N2 indices might be improper for interpreting the integrated spectra of galaxies because [N II] $\lambda 6583$ and $\mathrm{H} \alpha$ may arise not only in bona fide $\mathrm{H}$ II regions, but also in the diffuse ionized medium. Stasińska (2006) has recently proposed $\mathrm{Ar}_{3} \mathrm{O} 3^{5}$ and $\mathrm{S} 3 \mathrm{O} 3^{6}$ as new abundance indicators, which have the advantage of being unaffected by the chemical evolution effects. The advantages are superior to previous $N 2$ and $\mathrm{O} 3 \mathrm{~N} 2$ methods.

In short, one ideal metallicity indicator has to be monotonic and be independent of the internal reddenning and chemical evolution. Nagao et al. (2006) find that the $\mathrm{Ne} 3 \mathrm{O} 2$ index, i.e. $\log ([\mathrm{Ne} \mathrm{III}] \lambda 3869 /[\mathrm{O} \mathrm{II}] \lambda 3727)$, fulfills these requirements. They derive a relation of $\mathrm{Ne} 3 \mathrm{O} 2$ vs. $12+\log (\mathrm{O} / \mathrm{H})$ by using the Bayesian abundances provided by the MPA/JHU group ${ }^{7}$ for the metal rich galaxies in the SDSS (Tremonti et al. 2004) and the $T_{\mathrm{e}}$ abundances based on an electron temperature-sensitive line, [O III $] \lambda 4363$, for metal poor galaxies. The Bayesian metallicity could be problematic in some aspects, such as improper subtraction of the underlying starlight or unsuitable treatment of nitrogen enrichment in the HII galaxy model. As a result, there is a significant systematic difference between Bayesian metallicity and $T_{\mathrm{e}}$ metallicity (Yin et al. 2007), so it is necessary to recalibrate the $\mathrm{Ne} 3 \mathrm{O} 2$ index based on abundance derived from $T_{\mathrm{e}}$ method, which is believed to be the most reliable method to determine oxygen abundance. Also, the dispersion between [Ne III] $\lambda 33869 /[\mathrm{O}$ II $] \lambda 3727$ and oxygen abundance needs to be explained.

\footnotetext{
5 Ar3O3 = [Ar III $] \lambda 7135 /[\mathrm{O}$ III $] \lambda 5007$.

$6 \mathrm{~S} 3 \mathrm{O} 3=[\mathrm{S}$ III $] \lambda 9069 /[\mathrm{O}$ III $] \lambda 5007$.

7 See http://www.mpa-garching.mpg.de/SDSS/
} 
In this paper, we recalibrate the $\mathrm{Ne} 3 \mathrm{O} 2$ metallicity index based on a large sample of $\mathrm{H}$ II regions and $\mathrm{H}$ II galaxies where oxygen abundance is determined by the $T_{\mathrm{e}}$ method. For low metallicity galaxies (Sample I, see Sect. 2), we used a five-level statistical equilibrium model in the IRAF NEBULAR package (de Robertis et al. 1987; Shaw \& Dufour 1995), which makes use of the latest collision strengths and radiative transition probabilities to determine the $T_{\mathrm{e}}$ and $n_{\mathrm{e}}$. For high metallicity galaxies (Sample II, see Sect. 2), an empirical relation of $T_{\mathrm{e}}$ and strong spectral lines from Pilyugin (2001) was adopted for the electron temperature determination (their Eq. (11)). We also study the physical origin of the dispersion between $[\mathrm{Ne}$ III] $] \lambda 3869 /[\mathrm{O}$ II $] \lambda 3727$ and oxygen abundance.

The Sloan Digital Sky Survey (SDSS) is the most ambitious imaging and spectroscopic survey to date, and it will eventually cover a quarter of the sky (York et al. 2000). The large area coverage and moderately deep survey limit of the SDSS makes it suitable for studying the evolution of galaxies. Because of its homogeneity, the SDSS provides a large sample of H II galaxies where oxygen abundance can be calculated with the classic $T_{\mathrm{e}}$ method. The sample can be used to calibrate some metallicity indicators.

This paper is organized as follows. Based on an SDSS DR4 starbursts spectral sample and a sample of $\mathrm{HII}$ regions or H II galaxies from the literature, we present a sample to use for our metallicity index calibration (Sect. 2). In Sect. 3, we calibrate the Ne3O2 metallicity index. In Sect. 4, we study the origin of dispersion between oxygen abundance and $\mathrm{Ne} 3 \mathrm{O} 2$ index and check the accuracy of our calibration. In Sect. 5, we summarize the calibration result and discuss the merits of then $\mathrm{Ne} 3 \mathrm{O} 2$ indicator with respect to the other strong line indicators and then conclude this paper.

\section{Data sample}

The preferred method for determining oxygen abundances in galaxies is obtained from the ratio of auroral to nebular line intensities, such as [O III] $\lambda \lambda 4959,5007 /[\mathrm{O}$ III] $\lambda 4363$ (the so-called $T_{\mathrm{e}}$ method). In this paper, the adopted metallicities of H II regions and $\mathrm{H}$ II galaxies for calibration are determined from the $T_{\mathrm{e}}$ method.

We used H II galaxies from the Fourth Data Release (DR4) of the SDSS. After subtracting the underlying starlight using the method of Li et al. (2005) and Lu et al. (2006), we fit the emission line using the method of Dong et al. (2005). We made the internal reddening correction for the flux of all the emission line, using the two strongest Balmer lines, $\mathrm{H} \alpha / \mathrm{H} \beta$ and the effective absorption curve $\tau_{\lambda}=\tau_{V}(\lambda / 5500 \AA)^{-0.7}$, which was introduced by Charlot \& Fall (2000). Then, we made use of the spectral diagnostic diagrams from Kauffmann et al. (2003) to classify galaxies as starburst galaxies, active galactic nuclei (AGN), or unclassified. To reduce systematic and random errors from aperture effects, our galaxy samples are limited by the requirement that redshift $z>0.04$ (Kewley et al. 2005).

Within the primary starburst sample, two subsamples were selected from the SDSS-DR4 with the fluxes of spectral lines for all [Ne III] $\lambda 3869,[\mathrm{O}$ II $] \lambda 3727, \mathrm{H} \beta,[\mathrm{O}$ III] $\lambda 4959,[\mathrm{O}$ III $] \lambda 5007$, $\mathrm{H} \alpha$ and $[\mathrm{N}$ II] $\lambda 6583$ higher than 5 times the flux uncertainty. The difference of these two subsamples is that the first subsample (Sample I) was selected by the additional criterion from the [O III] $] \lambda 4363$ line, and the flux uncertainties for [O III] $] 4363$ was higher than $5 \sigma$. In all, 210 galaxies were included in this subsample. The [O III] $\lambda 4363$ is strongly dependent on the metallicity of galaxies and it becomes undetectable in high metallicity galaxies. Therefore, galaxies in Sample I are those with low metallicity. The electron temperature in the hightemperature zone $\left(T_{\mathrm{e}}(\mathrm{O} \mathrm{III})\right)$ of Sample I galaxies was calculated from [O III $] \lambda \lambda 4959,5007 /[\mathrm{O}$ III $] \lambda 4363$, using a five-level statistical equilibrium model in the IRAF NEBULAR package (de Robertis et al. 1987; Shaw \& Dufour 1995). In the second subsample (Sample II), galaxies have weak or no [O III] $\lambda 4363$ line, and 2960 galaxies were selected in this subsample containing generally metal rich galaxies. The $T_{\mathrm{e}}(\mathrm{O}$ III $)$ of the galaxies in Sample II was calculated with the empirical method following Pilyugin (2001) from the $R_{23}$ and $P$ parameter (their Eq. (11)).

To estimate the temperature in the low-temperature zone $T_{\mathrm{e}}(\mathrm{O}$ II $)$, the relation between $T_{\mathrm{e}}(\mathrm{O}$ II $)$ and $T_{\mathrm{e}}(\mathrm{O}$ III $)$ from Garnett (1992) are utilized:

$t_{\mathrm{e}}(\mathrm{O}$ II $)=0.7 \times t_{\mathrm{e}}(\mathrm{O}$ III $)+0.3$,

where $t_{\mathrm{e}}=T_{\mathrm{e}} / 10^{4} \mathrm{~K}$. After calculation of $T_{\mathrm{e}}$, we used the expressions from Izotov et al. (2006, their Eqs. (3) and (5)) to calculate the oxygen abundances. The average uncertainty from flux measurement in the computed $12+\log (\mathrm{O} / \mathrm{H})$ values is typically 0.10 dex in both samples.

To widen the oxygen abundance range, we built a large database of published reddening-corrected line fluxes for H II regions or H II galaxies, besides the H II galaxies from SDSS DR4. The data for H II regions in spiral galaxies in the literature with good [Ne III] $] 3869$, [O II] $] 3727$ and reliable $T_{\mathrm{e}}$ metallicity are taken from Garnett \& Kennicutt (1994), Garnett et al. (1997), Bresolin et al. (2004, 2005) and van Zee et al. (1998). The data for H II galaxies in the literature with very good signal-tonoise ratios are taken from Guseva et al. (2003a-c), Izotov et al. (1994, 1997, 1998, 2004,b, 2006), Lee et al. (2003a,b), Lee et al. (2004), Melbourne et al. (2004), Papaderos et al. (2006), and Thuan et al. (1995). In total, 84 H II regions of spiral galaxies and $446 \mathrm{H}$ II galaxies in the literature are adopted in our sample. The average uncertainty from flux measurement in the $12+\log (\mathrm{O} / \mathrm{H})$ values is typically within $0.10 \mathrm{dex}$.

\section{3. $\mathrm{Ne} 3 \mathrm{O} 2$ as a metallicity calibrator}

The upper panel of Fig. 1 shows the oxygen abundance as a function of $\mathrm{Ne} 3 \mathrm{O} 2$ for our samples. Apart from a few outliers in the SDSS DR4 sample, all the objects merge into a relatively tight, linear, and steep sequence, as expected. As expected from stellar nucleosynthesis (Izotov \& Thuan 1999) and confirmed by observation of low metallicity HII galaxies in the SDSS DR3 (Izotov et al. 2006), Ne and $\mathrm{O}$ are produced by the same stars, so the relationship between oxygen abundance and the $\mathrm{Ne} 3 \mathrm{O} 2$ index unlike $\mathrm{O} 3 \mathrm{~N} 2$ or $\mathrm{N} 2$ is not affected by the chemical evolution effects. The observed trend of $\mathrm{Ne} 3 \mathrm{O} 2$ is due to the fact that the ionization parameter has a strong metallicity dependence (Nagao et al. 2006). The two emission lines, [Ne III] $\lambda 3869$ and [O II] 33727 , have different degrees of ionization. Their ratio is strongly dependent on the ionization parameter and correlates with metallicity.

To confirm this view, we plot the relationship in Fig. 2 between $\mathrm{Ne} 3 \mathrm{O} 2$ index and the $P$ parameter for $\mathrm{H}$ II galaxies in SDSS DR4. The $P$ parameter is defined as ([O III $] \lambda \lambda 4959,5007) /$ ([O II $] \lambda 3727+[\mathrm{O}$ III $] \lambda \lambda 4959,5007)$ by Pilyugin $(2001)$, and it is a good representive of an ionization parameter. Figure 2 shows that the $\mathrm{Ne} 3 \mathrm{O} 2$ index is positive related to the $P$ parameter, which supports the idea that the $\mathrm{Ne} 3 \mathrm{O} 2$ index is negatively correlated with the metallicity, since more metal rich $\mathrm{H}$ II regions 

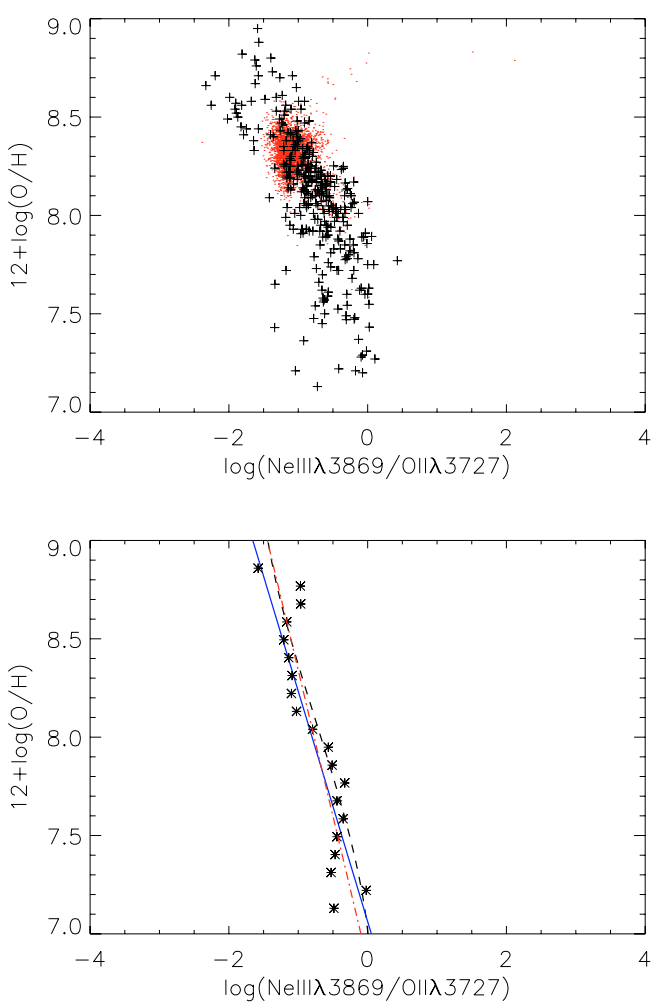

Fig. 1. Upper panel: $12+\log (\mathrm{O} / \mathrm{H})$ vs. $\log (\mathrm{Ne} 3 \mathrm{O} 2)$ for our data sample. Dots are galaxies from SDSS DR4. Crosses are from the literature. Lower panel: the solid line represents Eq. (2). Stars denote the mean for each bin of the oxygen abundance. The dot-dashed lines denote the best-fit function for each metallicity bin. The dashed curves are the calibration of Nagao et al. (2006).

are excited by a softer radiation field and have a lower ionization parameter. We have checked the relationship further between electron temperature $T_{\mathrm{e}}$ and $\mathrm{Ne} 3 \mathrm{O} 2$ index in Fig. 3 and find the observed trend of $\mathrm{Ne} 3 \mathrm{O} 2$ is partly due to the increase in $T_{\mathrm{e}}$ as metallicity decreases, which leads to an increase in the $\mathrm{Ne} 3 \mathrm{O} 2$ ratio. The comparison between Figs. 2 and 3 shows that, for the observed relation between $12+\log (\mathrm{O} / \mathrm{H})$ and the $\mathrm{Ne} 3 \mathrm{O} 2$ index, the contribution from the decrease in the ionization parameter $P$ with the increase in metallicity is stronger than the decrease in $T_{\mathrm{e}}$ with an increase in metallicity, since the $\mathrm{Ne} 3 \mathrm{O} 2$ index is more sensitive to the $P$ parameter than $T_{\mathrm{e}}$.

From the upper panel of Fig. 1, we can define a new metallicity calibration. The observed distribution of all the points in this figure is linear least-square-fitted by the following expression given as the solid line in the lower panel of Fig. 1:

$$
\begin{aligned}
12+\log (\mathrm{O} / \mathrm{H})= & -1.171( \pm 0.088) \times \mathrm{Ne} 3 \mathrm{O} 2 \\
& +7.063( \pm 0.727))
\end{aligned}
$$

with a standard error of 0.302 dex. In this panel, we also give the mean of the data with bin $=0.091 \mathrm{dex}$ in $12+\log (\mathrm{O} / \mathrm{H})$ (the stars) and the result of fitting the mean. We plot the calibration of Nagao et al. (2006) to compare with our result. In Sect. 4.2, we discuss why we prefer the fit for all the points and do not use the fit of mean.

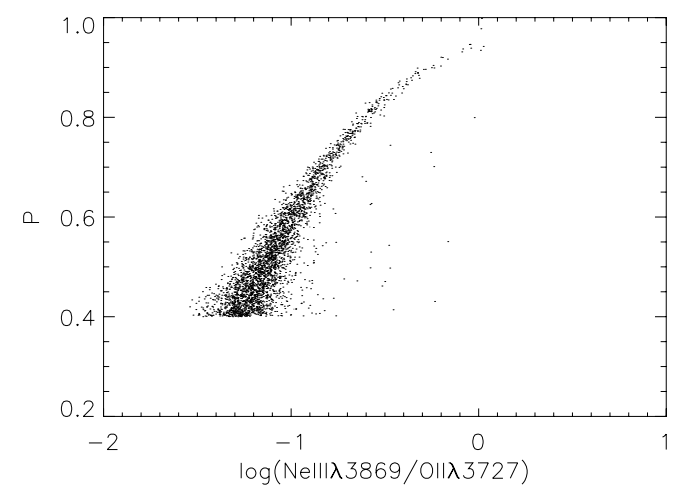

Fig. 2. The relationship between $\mathrm{Ne} 3 \mathrm{O} 2$ index and the $P$ parameter for H II galaxies in SDSS DR4. $P=([\mathrm{O}$ III $] \lambda 4959+[\mathrm{O}$ III $] \lambda 5007) /$ ([O II $] \lambda 3727+[\mathrm{O}$ III $] \lambda 4959+[\mathrm{O} \mathrm{III}] \lambda 5007)$.

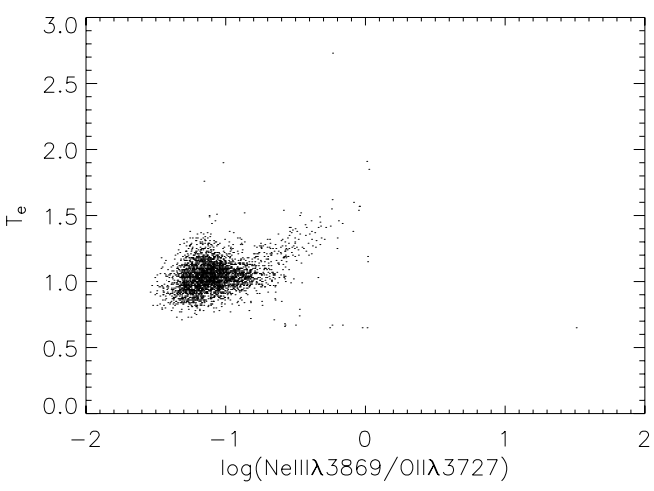

Fig. 3. The relationship between $\mathrm{Ne} 3 \mathrm{O} 2$ index and the electron temperature for HII galaxies in SDSS DR4. The electron temperature is in units of $10^{4} \mathrm{~K}$.

\section{Discussion}

\subsection{The origin of the dispersion}

Although the $T_{\mathrm{e}}$ metallicities are closely related to $\mathrm{Ne} 3 \mathrm{O} 2$ index, the dispersion of the relation is large, especially in the low metallicity region. Some of the scatter may come from the moderate depletion of oxygen onto grains in the metal rich galaxies. To show this view clearly, we calculated the $\log (\mathrm{Ne} / \mathrm{O})$ abundance ratios for Samples I and II using the method of Izotov et al. (2006) (their Eqs. (7) and (19)). Then we plotted the difference between $\log (\mathrm{O} / \mathrm{H})_{T_{\mathrm{e}}}-\log (\mathrm{O} / \mathrm{H})_{\mathrm{Ne} 3 \mathrm{O} 2}$ and the $\log (\mathrm{Ne} / \mathrm{O})$ abundance ratios in the upper panel of Fig. 4 for metal poor galaxies and the lower panel for metal rich galaxies. To compare Sample I and metal-poor emission-line galaxies in Izotov et al. (2006), we show the sample of Izotov et al. (2006) in the upper panel of Fig. 4.

As expected, Sample I is consistent with the sample of Izotov et al. (2006). There is a positive correlation between $\left(\log (\mathrm{O} / \mathrm{H})_{T_{\mathrm{e}}}\right)-\left(\log (\mathrm{O} / \mathrm{H})_{\mathrm{Ne} 3 \mathrm{O} 2}\right)$ and the $\log (\mathrm{Ne} / \mathrm{O})$ for metalpoor galaxies because more oxygen depletion would be present in the H II regions with higher metallicity (Izotov et al. 2006).

The lower panel of Fig. 4 shows more clearly that there is a clear correlation between $\log (\mathrm{O} / \mathrm{H})_{T_{\mathrm{e}}}-\log (\mathrm{O} / \mathrm{H})_{\mathrm{Ne} 3 \mathrm{O} 2}$ and the $\log (\mathrm{Ne} / \mathrm{O})$ for the metal rich galaxies because more oxygen is locked in the dust grains in the more metal-rich H II regions (Izotov et al. 2006). This correlation for Sample II can be given as a linear least-square fit:

$\log (\mathrm{O} / \mathrm{H})_{T_{\mathrm{e}}}-\log (\mathrm{O} / \mathrm{H})_{\mathrm{Ne} 3 \mathrm{O} 2}=0.610( \pm 0.024)$ $+0.999( \pm 0.037) \times \log (\mathrm{Ne} / \mathrm{O})$ 

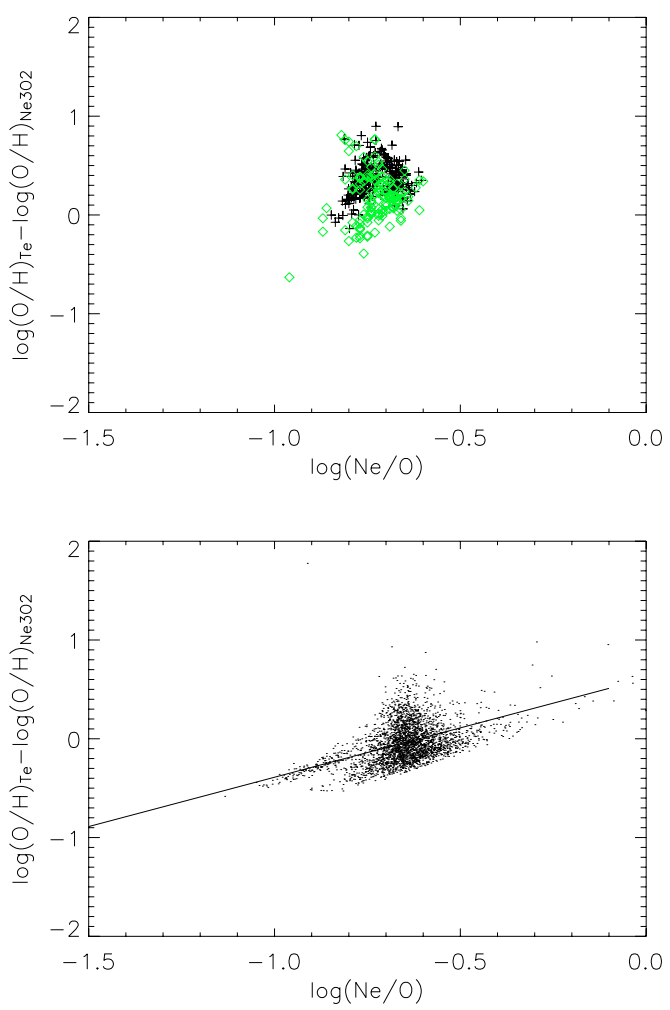

Fig. 4. Upper panel: correlation between $\log (\mathrm{O} / \mathrm{H})_{T_{\mathrm{e}}}-\log (\mathrm{O} / \mathrm{H})_{\mathrm{Ne} 3 \mathrm{O} 2}$ and the $\log (\mathrm{Ne} / \mathrm{O})$ abundance ratios of Sample I (crosses) and the sample of Izotov et al. (2006) (diamonds). $\log (\mathrm{O} / \mathrm{H})_{T_{\mathrm{e}}}-\log (\mathrm{O} / \mathrm{H})_{\mathrm{Ne} 3 \mathrm{O} 2}$ is the difference between $T_{\mathrm{e}}$ metallicity determined from the expressions in Izotov et al. (2006) and $\mathrm{Ne} 3 \mathrm{O} 2$ metallicity determined from Eq. (2). Lower panel: correlation between $\log (\mathrm{O} / \mathrm{H})_{T_{\mathrm{e}}}-\log (\mathrm{O} / \mathrm{H})_{\mathrm{Ne} 3 \mathrm{O} 2}$ and the $\log (\mathrm{Ne} / \mathrm{O})$ abundance ratios of Sample II (dots).

with a standard error of 0.104 dex. The corrected calibration of $\mathrm{Ne} 3 \mathrm{O} 2$ methods by considering this correlation will provide more accurate oxygen abundance, and the standard error of the corrected calibration in Fig. 1 will decrease to 0.198 dex, which is much less than the previous ones $(0.302 \mathrm{dex})$. This correlation can be used to correct the derived oxygen abundances from $\mathrm{Ne} 3 \mathrm{O} 2$ methods. However, to apply the $\mathrm{Ne} / \mathrm{O}$ correction to $12+\log (\mathrm{O} / \mathrm{H})$ abundances, we have to measure electron temperature $T_{\mathrm{e}}, \mathrm{H} \beta,[\mathrm{O} \mathrm{III}] \lambda 4959,[\mathrm{O} \mathrm{III}] \lambda 5007$, besides [Ne III] $\lambda 3869$ and $[\mathrm{O}$ II $] \lambda 3727$, so it will not always be practical.

\subsection{Comparison with others}

We plot the calibration of Nagao et al. (2006) to compare with Eq. (2) in the lower panel of Fig. 1. Some samples of both ours and Nagao's are selected randomly from the literature. As a result, the calibration might be affected by selection effects. To mitigate this effect, Nagao's calibration fits the mean in given metallicity bins. However, most of the SDSS data in our sample are concentrated in the metallicity range of $8.0<$ $12+\log (\mathrm{O} / \mathrm{H})<8.5$. As a result, if we fit the mean, the calibration will lose a lot of information in $8.0<12+\log (\mathrm{O} / \mathrm{H})<8.5$, because thousands of SDSS data are represented by several different mean values. As a result, the calibration fitting the mean will induce larger errors. In considering this, we used the calibration fitting all data points in the lower panel of Fig. 1 to compare with Nagao's calibration.

The lower panel of Fig. 1 shows that the oxygen abundance from Nagao's calibration is systematically higher than our

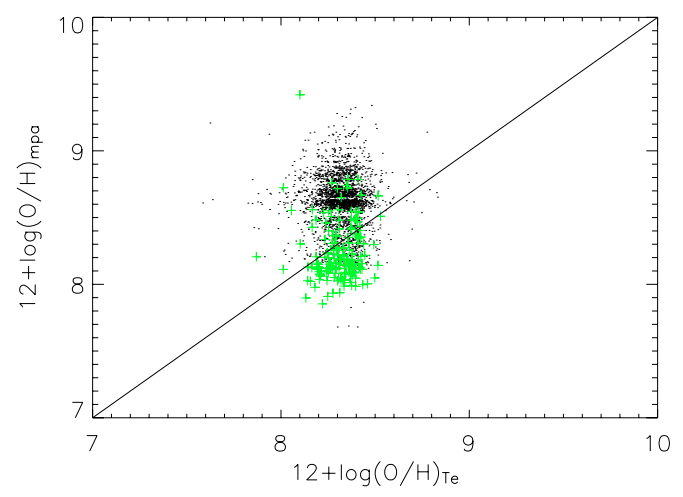

Fig. 5. Comparison of the $T_{\mathrm{e}}$ metallicities and Bayesian metallicities. Crosses are Sample I. Small dots are Sample II.

calibration at the given $\mathrm{Ne} 3 \mathrm{O} 2$, except that at the low metallicity end, our calibration is consistent with Nagao's because both ours and Nagao's abundances at the low metallicity end are derived by the $T_{\mathrm{e}}$ method. The difference between Nagao's calibration and ours in the high metallicity region could be caused by the oxygen abundances of most galaxies in the Nagao's sample being calculated by the Bayesian methods, while the oxygen abundances of our sample are all derived with the $T_{\mathrm{e}}$ methods. The origin of the difference between the Bayesian and $T_{\mathrm{e}}$ metallicities have already been discussed by Yin et al. (2007). They find that, for almost half of the sample galaxies (227 among 531 galaxies with $T_{\mathrm{e}}$ measurements), Bayesian metallicities are overestimated by a factor of about 0.34 dex on average, which is consistent with our result. They propose that the overestimates of Bayesian metallicities may be related to the onset of secondary $\mathrm{N}$ enrichment in models. Another reason for the lower $T_{\mathrm{e}}$ metallicities than Bayesian metallicities is that the [O III] $\lambda 4363$ emission line is biased by the very hot $\mathrm{H}$ II regions in each galaxy; thus, the global average temperature might be overestimated to $1000-3000 \mathrm{~K}$, which results in systematic underestimation of the oxygen abundance of $0.05-0.2 \mathrm{dex}$, as Nagao et al. (2006) propose.

In Fig. 5, we compare the $T_{\mathrm{e}}$ and Bayesian metallicities in our SDSS sample. It shows that the Bayesian metallicities of nearly half of the Sample II, where $T_{\mathrm{e}}$ are derived from the empirical method in Pilyugin (2001), are $\sim 0.3$ dex higher than $T_{\mathrm{e}}$ metallicity. The relationship between the $T_{\mathrm{e}}$ and Bayesian metallicities behaves very much like the result in Yin et al. (2007) (their Fig. 2), which supports the empirical $T_{\mathrm{e}}$ determination by Pilyugin (2001). This is consistent with $T_{\mathrm{e}}$ derived by the classic temperature-sensitive line. Similar evidence can be found in Shi et al. (2006) (their Fig. 2) where $T_{\mathrm{e}}$ metallicities from the empirical $T_{\mathrm{e}}$ determination have nearly the same relation with other strong line metallicities as do those from $T_{\mathrm{e}}$ derived from the classic temperature-sensitive line.

Generally speaking, all the methods should result in the same abundance value for a given nebula, but this is not the case in practice. It is evident that this discrepancy is caused by the problems both with models of HII regions and calibration. Why is the calibration of some types, such as the $T_{\mathrm{e}}$ method in this paper, regarded as the most reliable way to derive the oxygen abundance?

When comparing the numerical HII region models from Charlot \& Longhetti (2001), which are the basis of the Bayesian abundances, with the numerical models of other authors (Stasińska \& Leitherer 1996; McGaugh 1991; CLOUDY by Ferland et al. 1998; or Kewley \& Dopita 2002), one finds that there is significant disagreement between them because 


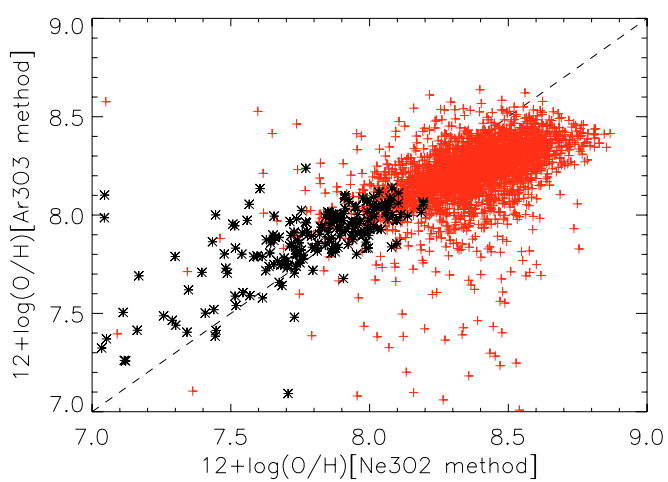

Fig. 6. Comparison of the $\mathrm{Ne} 3 \mathrm{O} 2$ and $\mathrm{Ar} 3 \mathrm{O} 3$ methods. $\mathrm{Ar} 3 \mathrm{O} 3$ metallicity is calculated using the calibration of Stasińska (2006). Ne3O2 metallicity is calculated using Eq. (2). Sample I is indicated by stars, and Sample II is indicated by crosses.

these models use different atomic data or different assumptions, and the stellar evolutionary synthesis code and photoionization code used in these models continuously improved. As a result, the present-day models cannot provide any uniform oxygen abundances.

As for calibrations, the calibrations based on the measurements of real H II regions is believed to be the most favorable way to derive the oxygen abundances, because it is calibrated by single $\mathrm{H}$ II region and will not be biased by other hot $\mathrm{H}$ II regions. That is why we use empirical $T_{\mathrm{e}}$ metallicity rather than Bayesian metallicity in high metallicity regions to calibrate $\mathrm{Ne} 3 \mathrm{O} 2$ index. There is a problem that the amount of $\mathrm{H}$ II regions with accurate measurements (a number of calibrating points) are not numerous enough. Therefore one has to use extrapolation, which can be a cause of uncertainty in the abundance determination.

Because Ar and Ne are produced by the same star, as expected by stellar nucleosynthesis (Izotov \& Thuan 1999), it is instructive to compare the Ne3O2- and Ar3O3-methods (Stasińska 2006). We show this comparison for SDSS DR4 sample in Fig. 6.

Although having a large scatter and a clear tendency of the observed relation to slightly deflect from $1: 1$, there is agreement between the oxygen abundance from the Ne3O2-method and the Ar3O3-method for most galaxies. The dispersion between the $T_{\mathrm{e}}$-based and Ar3O3 methods is especially large in the middle metallicity region $(7.95<12+\log (\mathrm{O} / \mathrm{H})<8.2)$. It may be caused by the fact that, when calibrating Ar3O3 index, the oxygen abundance is calculated using the empirical $P$-method, not the $T_{\mathrm{e}}$-based method (Stasińska 2006), and the empirical $P$-method is problematic in the middle metallicity region, especially $7.95<12+\log (\mathrm{O} / \mathrm{H})<8.2$ (Pilyugin 2001). The different oxygen abundance determination methods and different calibration methods may be the cause of the slope of the relation slightly deflecting from $1: 1$.

The accuracy of the $\mathrm{Ar} 3 \mathrm{O} 3, \mathrm{Ne} 3 \mathrm{O} 2$ indicators are comparable, as can be judged from Fig. 6. The advantage of the Ne302 method over the Ar303 one is that it does not demand a reliable reddening correction. The most prominent advantage of the Ne3O2 method over other metallicity indicators, such as the $R_{23^{-}}, N 2-, P-, \mathrm{O} 3 \mathrm{~N} 2-$ methods, lies in its ability to determine the oxygen abundance for high redshift galaxies. The Ne3O2 line ratio can be measurable at the extreme redshift limit of groundbased optical surveys. The $\mathrm{Ne} 3 \mathrm{O} 2, R_{23}, N 2, P, \mathrm{O} 3 \mathrm{~N} 2$ line ratios can be detected within several NIR atmospheric windows for the specific redshift $z \sim 1.5$ (Maier et al. 2006); but when it comes to the $z \sim 5-6$ galaxy, only the $\mathrm{Ne} 3 \mathrm{O} 2$ line ratio can be observed with near-infrared instruments. When it comes to the $z \sim 12$ galaxy, the $\mathrm{Ne} 3 \mathrm{O} 2$ line ratio can be detected with the James Webb Space Telescope (JWST), which uses a Near Infrared Camera and a Near Infrared Spectrometer (NIRSpec).

\section{Conclusions}

From the literature and SDSS DR4, we collected a large sample of spectroscopic measurements of $\mathrm{H}$ II regions and $\mathrm{H}$ II galaxies covering a wide range in metallicity $(7.0<12+\log (\mathrm{O} / \mathrm{H})<$ 9.0). The $T_{\mathrm{e}}$ values for 210 galaxies (Sample I) in SDSS DR4 are calculated with direct methods and 2960 galaxies (Sample II) with an empirical method. We confirm the existence of the correlation between $\mathrm{Ne} 3 \mathrm{O} 2$ index and oxygen abundance and have obtained a calibration that can be used as a method for determining oxygen abundances. The dispersion between oxygen abundance and the $\mathrm{Ne} 3 \mathrm{O} 2$ index may come partly from the moderate depletion of oxygen onto grains.

Though the Ne3O2 method is an empirical abundance determination method, we believe that also using the $\mathrm{Ne} 3 \mathrm{O} 2$ index as a metallicity calibrator presents several advantages:

1. The $\mathrm{Ne} 3 \mathrm{O} 2$ versus metallicity relation is monotonic.

2. The relationship between $\mathrm{Ne} 3 \mathrm{O} 2$ and metallicity is not affected by the chemical evolution effects because the $\mathrm{Ne}$ and $\mathrm{O}$ are produced by the same stars.

3. The Ne3O2 line ratio relies on the ratios of emission lines that are close in wavelength so do not depend on reddening corrections.

4. This $\mathrm{Ne} 3 \mathrm{O} 2$ line ratio can be measured for galaxies up to $z \sim 1.6$ using an optical telescope, up to $z \sim 5.2$ using nearinfrared instruments on the ground-based facilities, and up to $z \sim 12$ using JWST/NIRSpec; therefore, this flux ratio is a promising tool for metallicity studies at high redshift.

Acknowledgements. This work was supported by the Chinese National Science Foundation (Nos. 10521001, 10403006 and 10433010) and the National Basic Research Program of China (973 Program) No.2007CB815404. We thank G. Stasińska and L. S. Pilyugin for their helpful comments and suggestions. We are grateful to the AGN group at the Center for Astrophysics, University of the Science of Technology of China for processing the SDSS spectra for continuum decomposition and line fitting using the spectral analysis algorithm developed by the group. Funding for the Sloan Digital Sky Survey (SDSS) has been provided by the Alfred P. Sloan Foundation, the Participating Institutions, the National Aeronautics and Space Administration, the National Science Foundation, the US Department of Energy, the Japanese Monbukagakusho, and the Max Planck Society. The SDSS Web site is http://www.sdss.org/. The SDSS is managed by the Astrophysical Research Consortium (ARC) for the Participating Institutions. The Participating Institutions are The University of Chicago, Fermilab, the Institute for Advanced Study, the Japan Participation Group, The Johns Hopkins University, the Korean Scientist Group, Los Alamos National Laboratory, the Max-Planck-Institute for Astronomy (MPIA), the Max-PlanckInstitute for Astrophysics (MPA), New Mexico State University, University of Pittsburgh, University of Portsmouth, Princeton University, the United States Naval Observatory, and the University of Washington.

\section{References}

Bresolin, F., Garnett, D. R., \& Kennicutt, R. C., Jr. 2004, ApJ, 615, 228 Bresolin, F., Schaerer, D., González Delgado, R. M., \& Stasińska, G. 2005, A\&A, 441, 981

Charlot, S., \& Fall, S. M. 2000, ApJ, 539, 718C

Charlot, S., \& Longhetti, M. 2001, MNRAS, 323, 887

Cid Fernandes, R., Mateus, A., Sodré, L., Stasińska, G., \& Gomes, J. M. 2005, MNRAS, 358, 363

Contini, T., Treyer, M. A., Sullivan, M., \& Ellis, R. S. 2002, MNRAS, 330, 75

Denicoló, G., Terlevich, R., \& Terlevich, E. 2002, MNRAS, 330, 69

Dong, X.-B., Zhou, H.-Y., Wang, T.-G., et al. 2005, ApJ, 620, 629

Ferland, G. J., Korista, K. T., Verner, D. A., et al. 1998, PASP, 110, 761

Garnett, D. R., \& Kennicutt, R. C., Jr. 1994, ApJ, 426, 123 
Garnett, D. R., Shields, G. A., Skillman, E. D., Sagan, S. P., \& Dufour, R. J. 1997, ApJ, 489, 63

Guseva, N. G., Papaderos, P., Izotov, Y. I., et al. 2003a, A\&A, 407, 75

Guseva, N. G., Papaderos, P., Izotov, Y. I., et al. 2003b, A\&A, 407, 91

Guseva, N. G., Papaderos, P., Izotov, Y. I., et al. 2003c, A\&A, 407, 105

Izotov, Y. I., \& Thuan, T. X. 1998, ApJ, 500, 188

Izotov, Y. I., \& Thuan, T. X. 1999, ApJ, 511, 639

Izotov, Y. I., \& Thuan, T. X. 2004, ApJ, 602, 200

Izotov, Y. I., Thuan, T. X., \& Lipovetsky, V. A. 1994, ApJ, 435, 647

Izotov, Y. I., Thuan, T. X., \& Lipovetsky, V. A. 1997, ApJS, 108, 1

Izotov, Y. I., Papaderos, P., Guseva, N. G., Fricke, K. J., \& Thuan, T. X. 2004,

$$
\text { A\&A, 421, } 539
$$

Izotov, Y. I., Stasińska, G., Meynet, G., Guseva, N. G., \& Thuan, T. X. 2006, A\&A, 448, 955

Kauffmann, G., et al. 2003, MNRAS, 346, 1055

Kennicutt, R. C. 1998, ARA\&A, 36, 189

Kennicutt, R. C., Bresolin, F., \& Garnett, D. R. 2003, ApJ, 591, 801

Kewley, L. J., \& Dopita, M. A. 2002, ApJS, 142, 35

Kewley, L. J., Jansen, R. A., \& Geller, M. J. 2005, PASP, 117, 227

Lee, H., McCall, M. L., Kingsburgh, R. L., Ross, R., \& Stevenson, C. C. 2003a, AJ, 125,146

Lee, H., Grebel, E. K., \& Hodge, P. W. 2003b, A\&A, 401, 141

Lee, J. C., Salzer, J. J., \& Melbourne, J. 2004, ApJ, 616, 752

Li, C., Wang, T. G., Zhou, H.-Y., et al. 2005, AJ, 129, 669
Liang, Y. C., Yin, S. Y., Hammer, F., et al. 2006, ApJ, 652, 257

Lu, H., Zhou, H., Wang, J., et al. 2006, AJ, 131, 790

Maier, C., Lilly, S. J., Carollo, C. M., et al. 2006, ApJ, 639, 858

McGaugh, S. S. 1991, ApJ, 380, 140

Melbourne, J., Phillips, A., Salzer, J. J., Gronwall, C., \& Sarajedini, V. L. 2004, AJ, 127, 686

Nagao, T., Maiolino, R., \& Marconi, A. 2006, A\&A, 459, 85

Pagel, B. E. J., Edmunds, M. G., Blackwell, D. E., et al. 1979, MNRAS, 189, 95 Papaderos, P., Izotov, Y. I., Guseva, N. G., Thuan, T. X., \& Fricke, K. J. 2006, A\&A, 454, 119

Pettini, M., \& Pagel, B. E. J. 2004, MNRAS, 348, L59

Pilyugin, L. S. 2001, A\&A, 369, 594

de Robertis, M. M., Dufour, R. J., \& Hunt, R. W. 1987, JRASC, 81, 195

Shaw, R. A., \& Dufour, R. J. 1995, PASP, 107, 896

Shi, F., Kong, X., \& Cheng, F. Z. 2006, A\&A, 453, 487

Stasińska, G. 2005, A\&A, 434, 507

Stasińska, G. 2006, A\&A, 454, L127

Stasinska, G., \& Leitherer, C. 1996, ApJS, 107, 661

Thuan, T. X., Izotov, Y. I., \& Lipovetsky, V. A. 1995, ApJ, 445, 108

Tremonti, et al. 2004, ApJ, 613, 898

van Zee, L., Salzer, J. J., Haynes, M. P., O’Donoghue, A. A., \& Balonek, T. J. 1998, AJ, 116, 2805

Yin, S. Y., Liang, Y. C., Hammer, F., et al. 2007, A\&A, 462, 535

York, D. G., Hall, P. B., Vanden Berk, D. E., et al. 2001, AJ, 122, 549 